\title{
Optimal Transmission Policies over Vector Gaussian Broadcast Channels with Energy Harvesting Transmitters
}

\author{
Omur Ozel $^{1}$, Jing Yang ${ }^{2}$, and Sennur Ulukus ${ }^{1}$ \\ ${ }^{1}$ Department of Electrical and Computer Engineering, University of Maryland, College Park, MD 20742 \\ ${ }^{2}$ Department of Electrical and Computer Engineering, University of Wisconsin-Madison, Madison, WI 53706
}

\begin{abstract}
We consider an energy harvesting transmitter sending messages to two users over a vector broadcast channel. Energy required for communication arrives (is harvested) at the transmitter and a finite-capacity battery stores it before being consumed for transmission. Under off-line knowledge of energy arrivals, we obtain the trade-off between the performances of the users by characterizing the maximum departure region in a given interval. We show that the optimal total transmit power policy that achieves the boundary of the maximum departure region is the same as the optimal policy for the scalar singleuser channel, which does not depend on the priorities of the users. The optimal total transmit power is found by a directional water-filling algorithm and the optimal splitting of the power among the users and the parallel channels is performed in each epoch separately.
\end{abstract}

\section{INTRODUCTION}

A distinct characteristic of energy harvesting communication systems is that the energy required for communication arrives during the session in which communication takes place. The transmitter is able to harvest energy from nature in order to recharge its battery. The energy is modeled to arrive (be harvested) at arbitrary instants and in arbitrary amounts. Therefore, transmission schemes must adapt to the incoming energy. In this paper, we consider communication with an energy harvesting transmitter over a vector Gaussian broadcast channel.

In particular, we consider an energy harvesting transmitter that sends data over a vector Gaussian broadcast channel as in Fig. 1. Data for the two receivers are backlogged at the transmitter buffers while arriving energy is stored in a finitecapacity battery. Energy arrivals are known by the transmitter a priori. The transmitter has to adapt its transmission power with respect to the available energy and also avoid possible energy overflows due to the finite-capacity battery.

There has been recent research effort on understanding data transmission with an energy harvesting transmitter that has a rechargeable battery [1]-[7]. In [1], data transmission with energy harvesting sensors is considered and optimal online policy for controlling admissions into the data buffer is derived using a dynamic programming framework. In [2], energy management policies which stabilize the data queue

This work was supported by NSF Grants CCF 07-29127, CNS 09-64632, CCF 09-64645, CCF 10-18185.

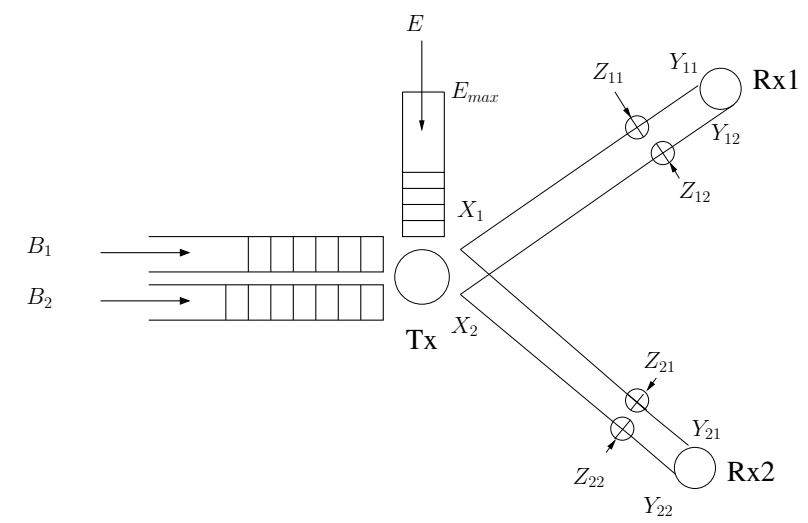

Fig. 1. The two-user vector broadcast channel with an energy harvesting transmitter.

are proposed for single-user communication and some delay optimality properties are derived under a linearity assumption for the power-rate relation. In [3], throughput optimal energy allocation is studied for energy harvesting systems in a time constrained slotted setting. In [4], [5], minimization of the transmission completion time is considered in an energy harvesting single-user system and the optimal solution is obtained analytically and using a geometric algorithm. In [6], energy harvesting transmitters with batteries of finite energy storage capacity are considered and the problem of throughput maximization by a deadline is solved in a static channel. In [7], optimal off-line and on-line transmission policies are given for a single-user energy harvesting transmitter operating in a fading channel. In [8] and [9], optimal off-line policies are developed for the static AWGN broadcast channel with an infinite capacity battery, concurrently and independently. References [10], [11] extend the broadcasting framework to the case of a finite-capacity battery energy harvesting transmitter.

In this paper, we extend the line of research on off-line optimal scheduling in energy harvesting communication systems to vector Gaussian broadcast channels. We characterize the trade-off between the performances of the users by obtaining the maximum departure region [8], [11] by a deadline $T$ and determine the optimal off-line policies. We prove that the time sequence of the total allocated power in each epoch is the same as the allocation in a scalar single-user channel, independent 
of the priorities of the receivers. The power is allocated to each channel and each user separately for each epoch according to the strength order of the users using the waterfilling algorithm described in [12], [13].

\section{Two-User Vector GAUSSiAn Broadcast CHANNEL}

In a two-user vector Gaussian broadcast channel, one transmitter sends data to two receivers over independent parallel channels. The model is depicted in Fig. 1. We consider the case where there are two parallel channels only. The generalization to more than two parallel channels is straightforward, and left out for brevity and clarity of presentation in this paper.

\section{A. Channel Model}

The received signals at the two receivers are

$$
\begin{aligned}
Y_{1 i} & =X_{i}+Z_{1 i}, & i & =1,2 \\
Y_{2 i} & =X_{i}+Z_{2 i}, & i & =1,2
\end{aligned}
$$

where $X_{i}$ is the signal transmitted in the $i$ th parallel channel, and $Z_{1 i}$ and $Z_{2 i}$ are Gaussian noises with variances $\sigma_{1 i}^{2}$ and $\sigma_{2 i}^{2}$, respectively. If $\sigma_{1 i}^{2} \leq \sigma_{2 i}^{2}$ for all $i$, or $\sigma_{2 i}^{2} \leq \sigma_{1 i}^{2}$ for all $i$, then the overall channel is degraded in favor of user 1 or user 2, respectively, and hence the problem has the same structure as the scalar broadcast channel problem. Therefore, we consider the case $\sigma_{11}^{2}<\sigma_{21}^{2}$ and $\sigma_{12}^{2}>\sigma_{22}^{2}$ where the overall broadcast channel is not degraded.

Assuming that the transmitter transmits with power $P$, the achievable rate region for this two-user parallel broadcast channel is [12], [13]

$$
\begin{aligned}
R_{1} \leq & \frac{1}{2} \log _{2}\left(1+\frac{\alpha_{1} \beta P}{\sigma_{11}^{2}}\right) \\
& +\frac{1}{2} \log _{2}\left(1+\frac{\alpha_{2}(1-\beta) P}{\left(1-\alpha_{2}\right)(1-\beta) P+\sigma_{12}^{2}}\right) \\
R_{2} \leq & \frac{1}{2} \log _{2}\left(1+\frac{\left(1-\alpha_{2}\right)(1-\beta) P}{\sigma_{22}^{2}}\right) \\
& +\frac{1}{2} \log _{2}\left(1+\frac{\left(1-\alpha_{1}\right) \beta P}{\alpha_{1} \beta P+\sigma_{21}^{2}}\right)
\end{aligned}
$$

where $\beta P$ is the power allocated to the first parallel channel, and $(1-\beta) P$ is the power allocated to the second parallel channel, $\alpha_{1}$ and $\alpha_{2}$ are the fractions of powers spent for the message transmitted to user 1 in each parallel channel. By varying $\alpha_{1}, \alpha_{2}$ and $\beta$, we obtain a family of achievable regions and their union is the capacity region. Any operating point on the boundary of the capacity region is fully characterized by solving for the power allocation policy that maximizes $\mu_{1} R_{1}+$ $\mu_{2} R_{2}$ for some $\left(\mu_{1}, \mu_{2}\right)$. For any $\mu_{1}, \mu_{2}$, there exist $P^{*}, \alpha_{1}^{*}, \alpha_{2}^{*}$ and $\beta^{*}$ that achieve the corresponding point on the boundary of the capacity region [12], [13].

\section{B. Energy and Power-Rate Models}

In a two-user energy harvesting vector broadcast channel, the transmitter has three queues as in Fig. 1: two data queues where data packets for the two receivers are stored, and an energy queue where the arriving (harvested) energy is stored.
The energy queue, i.e., the battery, can store at most $E_{\max }$ units of energy, which is used for transmission only, i.e., energy required for processing is not considered.

We consider an off-line setting where energy arrivals that occur throughout the communication are known by the transmitter a priori. Performance of any transmission policy with a priori knowledge provides an upper bound for that of a real time system. In the interval $[0, T]$, there are $N$ energy arrivals at times $\left\{s_{k}\right\}, k=1, \ldots, N$ as in Fig. 2. As a convention, we let $s_{0}=0$ and $s_{N+1}=T$. We call the time between the energy arrivals as epoch and thus $\left[s_{k-1}, s_{k}\right)$ is the $k$ th epoch. The length of the $k$ th epoch is denoted as $\ell_{k}=s_{k}-s_{k-1}$. In the $[0, T]$ interval, there are $N+1$ epochs.

Whenever an input signal $x$ is transmitted with power $p$ in an epoch of duration $\ell, R_{1} \ell$ and $R_{2} \ell$ bits of data are served out from the backlogs of receivers 1 and 2 at the transmitter, with the cost of $p \ell$ units of energy depletion from the energy queue. Here, $\left(R_{1}, R_{2}\right)$ is the rate allocation for this epoch. $\left(R_{1}, R_{2}\right)$ must reside in the corresponding capacity region, i.e., $\left(R_{1}, R_{2}\right)$ must satisfy (3) and (4). Extending this for continuous time, if at time $t$ the transmit power is $P(t)$, the instantaneous rate pairs $\left(R_{1}(t), R_{2}(t)\right)$ reside in the corresponding capacity region.

Power policy is constrained due to the following reasons: energy arrivals at arbitrary times and finite battery storage capacity. Let $h_{-}(t)=\max \left\{i: s_{i}<t\right\}$. As energy that has not arrived yet cannot be used at the current time, there is a causality constraint on the power policy $P(t)$ :

$$
\int_{0}^{t} P(\tau) d \tau \leq \sum_{i=0}^{h_{-}(t)} E_{j}, \quad \forall t \in[0, T]
$$

Moreover, if the energy level in the battery exceeds $E_{\max }$, some energy overflows and hence lost without being utilized. In order to guarantee no-energy-overflow throughout the duration of communication, it suffices to ensure that the energy level in the battery never exceeds $E_{\max }$ at the times of energy arrivals. Hence, we have the following no-energy-overflow constraint:

$$
\int_{0}^{t} P(\tau) d \tau \geq\left(\sum_{i=0}^{h_{+}(t)} E_{i}-E_{\max }\right)^{+}, \quad \forall t \in[0, T]
$$

where $h_{+}(t)=\max \left\{i: s_{i} \leq t\right\}$. The constraint in (6) imposes that at least $\sum_{i=0}^{k} E_{i}-E_{\max }$ amount of energy has been consumed by the time the $k$ th energy arrives so that the battery can accommodate $E_{k}$ at time $s_{k}$.

\section{MAXIMUm Departure Region}

We now characterize the trade-off between the performances of user 1 and user 2 by finding the region of bits sent for receivers 1 and 2 in the interval $[0, T]$ with off-line knowledge of energy arrivals. The number of bits sent for users 1 and 2 are:

$$
B_{1}=\int_{0}^{T} R_{1}(\tau) d \tau, \quad B_{2}=\int_{0}^{T} R_{2}(\tau) d \tau
$$




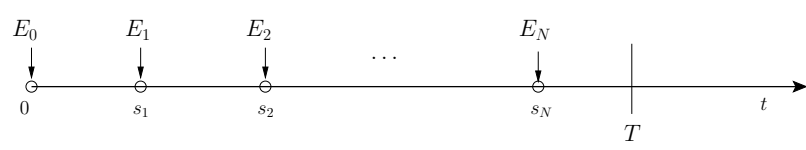

Fig. 2. The energy arrival sequence.

Definition 1 For any fixed transmission duration $T$, the maximum departure region, denoted as $\mathcal{D}(T)$, is the union of $\mathcal{R}\left(B_{1}, B_{2}\right)=\left\{\left(b_{1}, b_{2}\right): 0 \leq b_{1} \leq B_{1}, 0 \leq b_{2} \leq B_{2}\right\}$ where $\left(B_{1}, B_{2}\right)$ is the total number of bits sent by some power policy that satisfies energy causality (5) and no-energy-overflow (6) conditions over the duration $[0, T)$.

We have the following lemma, the proof of which can be carried out following the proofs of Lemma 2 in [8] and Lemma 1 in [11] and hence is skipped here for brevity.

Lemma $1 \mathcal{D}(T)$ is a convex region.

The instantaneous rates $r_{1}(t)$ and $r_{2}(t)$ allocated for users 1 and 2 are determined as a function of the instantaneous power, $P(t)$, power share of the 1st channel, $\beta(t)$, and the power shares of user 1 in the $i$ th channel, $\alpha_{i}(t), i=1,2$, via (3) and (4). The instantaneous power, $P(t)$, is subject to the energy causality and no-energy-overflow conditions in (5) and (6), respectively.

Due to the convexity of $\mathcal{D}(T)$ in Lemma 1 and the concave power-rate relation, an optimal policy should remain constant in any epoch (c.f. Lemma 1 in [8] and Lemma 2 in [4], [5]). Therefore, we consider a power policy as a sequence of powers allocated for each epoch $\left\{p_{i}\right\}_{i=1}^{N+1}$ with the 1st channel's share $\left\{\beta_{i}\right\}_{i=1}^{N+1}$, the power share of user 1 in each channel $\left\{\left(\alpha_{1 i}, \alpha_{2 i}\right)\right\}_{i=1}^{N+1}$. Then, the energy causality and noenergy-overflow conditions in (5) and (6) reduce to the following constraints, respectively, which are described by a finite sequence of powers:

$$
\begin{aligned}
& \sum_{i=1}^{k} p_{i} \ell_{i} \leq \sum_{i=0}^{k-1} E_{i}, \quad k=1, \ldots, N+1 \\
& \sum_{i=1}^{k} p_{i} \ell_{i} \geq\left(\sum_{i=0}^{k} E_{i}-E_{\max }\right)^{+}, \quad k=1, \ldots, N
\end{aligned}
$$

Here (8) is due to the energy causality constraint in (5) and (9) is due to the no-energy-overflow condition in (6). We define the following functions:

$$
\begin{array}{r}
r_{1}\left(\alpha_{1}, \alpha_{2}, \beta, p\right)=\frac{1}{2} \log _{2}\left(1+\frac{\alpha_{1} \beta p}{\sigma_{11}^{2}}\right) \\
+\frac{1}{2} \log _{2}\left(1+\frac{\alpha_{2}(1-\beta) p}{\left(1-\alpha_{2}\right)(1-\beta) p+\sigma_{12}^{2}}\right) \\
r_{2}\left(\alpha_{1}, \alpha_{2}, \beta, p\right)=\frac{1}{2} \log _{2}\left(1+\frac{\left(1-\alpha_{1}\right) \beta p}{\alpha_{1} \beta p+\sigma_{21}^{2}}\right) \\
+\frac{1}{2} \log _{2}\left(1+\frac{\left(1-\alpha_{2}\right)(1-\beta) p}{\sigma_{22}^{2}}\right)
\end{array}
$$

which are the rates achieved by users 1 and 2 , respectively, if $p$ is allocated to the channels with the first parallel channel's share $\beta p$, and user 1's power share $\left(\alpha_{1}, \alpha_{2}\right)$ in each channel. By Lemma 1, any point on the boundary of the maximum departure region $\mathcal{D}(T)$ can be characterized by solving the following optimization problem:

$$
\begin{aligned}
\max _{\boldsymbol{\alpha}_{1}, \boldsymbol{\alpha}_{2}, \boldsymbol{\beta}, \mathbf{p}} & \mu_{1} \sum_{i=1}^{N+1} r_{1}\left(\alpha_{1 i}, \alpha_{2 i}, \beta_{i}, p_{i}\right) \ell_{i} \\
& +\mu_{2} \sum_{i=1}^{N+1} r_{2}\left(\alpha_{1 i}, \alpha_{2 i}, \beta_{i}, p_{i}\right) \ell_{i} \\
\text { s.t. } \quad & \sum_{i=1}^{k} p_{i} \ell_{i} \leq \sum_{i=0}^{k-1} E_{i}, \quad \forall k \\
& \sum_{i=1}^{k} p_{i} \ell_{i} \geq\left(\sum_{i=0}^{k} E_{i}-E_{\max }\right)^{+}, \quad \forall k
\end{aligned}
$$

In (12), $\boldsymbol{\alpha}_{1}, \boldsymbol{\alpha}_{2}, \boldsymbol{\beta}, \mathbf{p}$ collectively denote the vector of total powers and power shares for the parallel channels and users. The optimization problem (12) is not a convex problem as the variables $p_{i}, \beta_{i}, \alpha_{1 i}$ and $\alpha_{2 i}$ appear in product forms in the expression of $r_{i}\left(\alpha_{1}, \alpha_{2}, \beta, p\right)$, causing it to be non-concave in $\alpha_{i}, p$ and $\beta$ jointly. However, for any given $\alpha_{1}, \alpha_{2}, \beta$ we note that $\mu_{1} r_{1}\left(\alpha_{1}, \alpha_{2}, \beta, p\right)+\mu_{2} r_{2}\left(\alpha_{1}, \alpha_{2}, \beta, p\right)$ is concave with respect to $p$.

The optimization problem in (12) can be cast as a sequence of optimization problems of the following form given the power $p$ :

$$
\begin{aligned}
\max _{\alpha_{1}, \alpha_{2}, \beta} & \mu_{1} r_{1}\left(\alpha_{1}, \alpha_{2}, \beta, p\right)+\mu_{2} r_{2}\left(\alpha_{1}, \alpha_{2}, \beta, p\right) \\
\text { s.t. } & 0 \leq \alpha_{1}, \alpha_{2}, \beta \leq 1
\end{aligned}
$$

Note that given $\beta$ and $p$, optimal $\alpha_{1}$ and $\alpha_{2}$ can be separately calculated. In particular, (13) is solved at $\alpha_{1}=\alpha_{1}^{*}(\beta, p)$ and $\alpha_{2}=\alpha_{2}^{*}(\beta, p)$. If $\frac{\mu_{2}}{\mu_{1}} \leq 1, \alpha_{1}^{*}(\beta, p)=1$ while if $\frac{\mu_{2}}{\mu_{1}} \geq \frac{\sigma_{21}^{2}}{\sigma_{11}^{2}}$, $\alpha_{1}^{*}(\beta, p)=0$ for all $\beta$. If $1<\frac{\mu_{2}}{\mu_{1}}<\frac{\sigma_{21}^{2}}{\sigma_{11}^{2}}$, we have

$$
\alpha_{1}^{*}(\beta, p)= \begin{cases}1, & 0 \leq p \leq \frac{\mu_{2} \sigma_{11}^{2}-\mu_{1} \sigma_{21}^{2}}{\beta\left(\mu_{1}-\mu_{2}\right)} \\ \frac{1}{\beta p} \frac{\mu_{2} \sigma_{11}^{2}-\mu_{1} \sigma_{21}^{2}}{\mu_{1}-\mu_{2}}, & p \geq \frac{\mu_{2} \sigma_{11}^{2}-\mu_{1} \sigma_{21}^{2}}{\beta\left(\mu_{1}-\mu_{2}\right)}\end{cases}
$$

Similarly, if $\frac{\mu_{1}}{\mu_{2}} \leq 1, \alpha_{2}^{*}(\beta, p)=0$ while if $\frac{\mu_{1}}{\mu_{2}} \geq \frac{\sigma_{12}^{2}}{\sigma_{22}^{2}}$ then $\alpha_{2}^{*}(\beta, p)=1$ for all $\beta$. If $1<\frac{\mu_{1}}{\mu_{2}}<\frac{\sigma_{12}^{2}}{\sigma_{22}^{2}}$,

$\alpha_{2}^{*}(\beta, p)= \begin{cases}0, & 0 \leq p \leq \frac{\mu_{1} \sigma_{22}^{2}-\mu_{2} \sigma_{12}^{2}}{(1-\beta)\left(\mu_{2}-\mu_{1}\right)} \\ 1-\frac{1}{(1-\beta) p} \frac{\mu_{1} \sigma_{22}^{2}-\mu_{2} \sigma_{12}^{2}}{\mu_{2}-\mu_{1}}, & p \geq \frac{\mu_{1} \sigma_{22}^{2}-\mu_{2} \sigma_{12}^{2}}{(1-\beta)\left(\mu_{2}-\mu_{1}\right)}\end{cases}$

Hence, (13) is equivalent to the following given $p$ :

$$
\max _{0 \leq \beta \leq 1} \mu_{1} r_{1}^{*}(\beta, p)+\mu_{2} r_{2}^{*}(\beta, p)
$$

where $r_{1}^{*}(\beta, p)=r_{1}\left(\alpha_{1}^{*}(\beta, p), \alpha_{2}^{*}(\beta, p), \beta, p\right)$ and $r_{2}^{*}(\beta, p)=$ $r_{2}\left(\alpha_{1}^{*}(\beta, p), \alpha_{2}^{*}(\beta, p), \beta, p\right)$. The solution of (16) has a waterfilling interpretation. Due to the optimal $\alpha_{1}$ and $\alpha_{2}$ in (14) 
and (15), if $\frac{\mu_{2}}{\mu_{1}} \leq \frac{\sigma_{22}^{2}}{\sigma_{12}^{2}}$, all the power is allocated to user 1 and the power is split over the parallel channels by a singleuser water-filling solution. Similarly, if $\frac{\mu_{2}}{\mu_{1}} \geq \frac{\sigma_{21}^{2}}{\sigma_{11}^{2}}$, then all the power is allocated for the second user and the power is split into two via single-user water-filling. In the remaining ranges of $\frac{\mu_{2}}{\mu_{1}}$, depending on the total power $p$, we have

$$
\begin{aligned}
\beta^{*} p & =\left(\mu_{u_{1}} \lambda-\sigma_{u_{1} 1}^{2}\right)^{+} \\
\left(1-\beta^{*}\right) p & =\left(\mu_{u_{2}} \lambda-\sigma_{u_{2} 2}^{2}\right)^{+}
\end{aligned}
$$

where $u_{1}, u_{2} \in\{1,2\}$ and $\beta^{*}$ is the optimizer of (16). In all of the cases, the water level is unique. This solution is equivalent to water-filling described in [12], [13]. The outcome of the optimization problem depends on the power $p$. Let us define the following function of $p$ :

$$
g(p)=\max _{0 \leq \beta \leq 1} \mu_{1} r_{1}^{*}(\beta, p)+\mu_{2} r_{2}^{*}(\beta, p)
$$

We have the following lemma which is proved in [14].

Lemma $2 g(p)$ is a concave function of $p$ for all $\mu_{1}, \mu_{2}$.

Using Lemma 2, as in [11], we obtain an important characteristic of the optimal policies that achieve the boundary of the maximum departure region $\mathcal{D}(T)$.

Lemma 3 For any $\left(\mu_{1}, \mu_{2}\right)$, the optimal total power allocation sequence is the same as the optimal scalar single-user power allocation sequence.

The scalar single-user power allocation is found using the directional waterfilling algorithm [7]. The directional waterfilling algorithm requires walls at the points of energy arrival, with right permeable water taps in each wall which allows at most $E_{\max }$ amount of water to flow, as shown in Fig. 3. First, the taps are kept off and transfer from one epoch to the other is not allowed. Each incoming energy $E_{i}$ is spread in the time interval between energy arrivals and the water level is initially $\frac{E_{i}}{\ell_{i}}$. Then, each right permeable tap is turned on one by one. If the water level in the $i-1$ st epoch is smaller than that of the $i$ th epoch, then some energy is transferred from the $i-1 \mathrm{st}$ epoch to the $i$ th epoch to equalize the water levels. At most $E_{\max }-E_{i}$ amount of energy can be transferred considering that $E_{i}$ amount of energy already flowed. The resulting water levels yield the total power sequence $p_{i}^{*}$.

With Lemma 3 and the preceding findings, we obtain the full structure of a point on the boundary of the maximum departure region $\mathcal{D}(T)$. We first calculate the total power allocated at each receiver using the directional waterfilling algorithm. As a result, we get the sequence of total powers allocated at each time epoch, $\left\{p_{i}^{*}\right\}_{i=1}^{N+1}$. Then, we divide each $p_{i}^{*}$ as $p_{i 1}^{*}$ and $p_{i 2}^{*}$ allocated to the two parallel broadcast channels by means of the waterfilling procedure described in equations (17)-(18). With this, we get $p_{i 1}^{*}$ and $p_{i 2}^{*}$ as well as the corresponding power shares of the receivers in each broadcast channel $\alpha_{1}^{*}\left(p_{i 1}^{*}\right)$ and $\alpha_{2}^{*}\left(p_{i 2}^{*}\right)$. Then, $\left(B_{1}^{*}, B_{2}^{*}\right)$ point
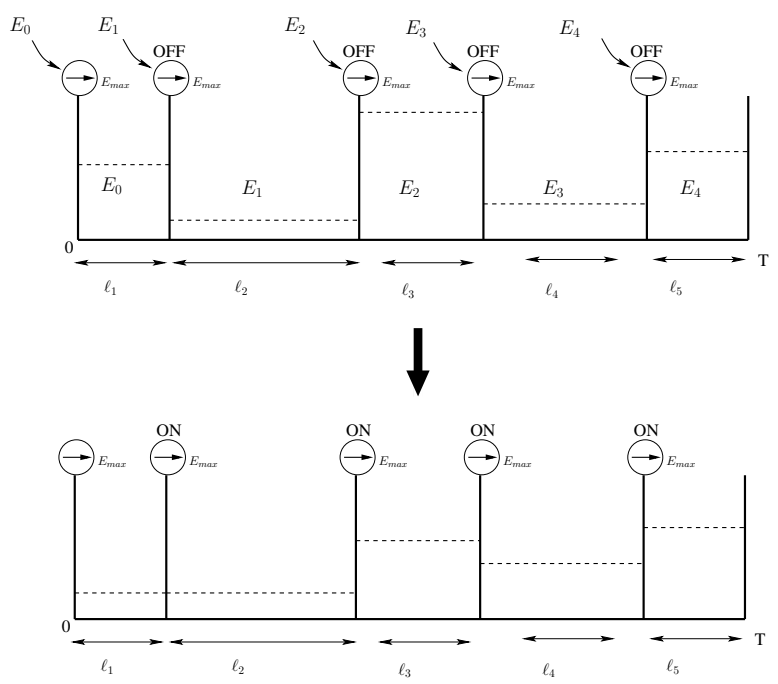

Fig. 3. Directional waterfilling algorithm.

that correspond to the priority coefficients $\mu_{1}^{*}$ and $\mu_{2}^{*}$ is

$$
\begin{aligned}
B_{1}^{*}=\sum_{i=1}^{K+1} \frac{1}{2} \log \left(1+\frac{\alpha_{1}^{*}\left(p_{i 1}^{*}\right) p_{i 1}^{*}}{\sigma_{11}^{2}}\right) \ell_{i} \\
+\frac{1}{2} \log \left(1+\frac{\left(1-\alpha_{2}^{*}\left(p_{i 2}^{*}\right)\right) p_{i 2}^{*}}{\alpha_{2}^{*}\left(p_{i 2}^{*}\right) p_{i 2}^{*}+\sigma_{21}^{2}}\right) \ell_{i} \\
B_{2}^{*}=\sum_{i=1}^{K+1} \frac{1}{2} \log \left(1+\frac{\alpha_{2}^{*}\left(p_{i 2}^{*}\right) p_{i 2}^{*}}{\sigma_{22}^{2}}\right) \ell_{i} \\
+\frac{1}{2} \log \left(1+\frac{\left(1-\alpha_{1}^{*}\left(p_{i 1}^{*}\right)\right) p_{i 1}^{*}}{\alpha_{1}^{*}\left(p_{i 1}^{*}\right) p_{i 1}^{*}+\sigma_{12}^{2}}\right) \ell_{i}
\end{aligned}
$$

\section{Numerical Results}

We consider a band-limited two-user AWGN broadcast channel with two parallel channels operating with a bandwidth of $W=1 \mathrm{MHz}$ and under noise power spectral density $N_{0}=10^{-19} \mathrm{~W} / \mathrm{Hz}$. In the first channel, the path loss between the transmitter and receiver 1 is $c_{11}=100 \mathrm{~dB}$ and between the transmitter and receiver 2 is $c_{21}=105 \mathrm{~dB}$. The second parallel channel has path loss coefficients $c_{12}=107 \mathrm{~dB}$ and $c_{22}=103 \mathrm{~dB}$ and the resulting rate expressions are

$$
\begin{aligned}
r_{1}=\log _{2} & \left(1+\frac{\alpha_{1} \beta P}{n_{11}}\right) \\
& +\log _{2}\left(1+\frac{\alpha_{2}(1-\beta) P}{\left(1-\alpha_{2}\right)(1-\beta) P+n_{12}}\right) \mathrm{Mbps} \\
r_{2}=\log _{2} & \left(1+\frac{\left(1-\alpha_{2}\right)(1-\beta) P}{n_{22}}\right) \\
& +\log _{2}\left(1+\frac{\left(1-\alpha_{1}\right) \beta P}{\alpha_{1} \beta P+n_{21}}\right) \text { Mbps }
\end{aligned}
$$

where $n_{11}=1, n_{21}=10^{0.5}, n_{12}=10^{0.7}, n_{22}=10^{0.3}$ and $P$ is in $\mathrm{mW}$. We assume that the battery capacity is $E_{\max }=10$ $\mathrm{mJ}$ and the energy arrivals occur at time instants $s_{1}=2 \mathrm{~s}$, $s_{2}=5 \mathrm{~s}, s_{3}=8 \mathrm{~s}, s_{4}=9 \mathrm{~s}, s_{5}=12 \mathrm{~s}$ with amounts 

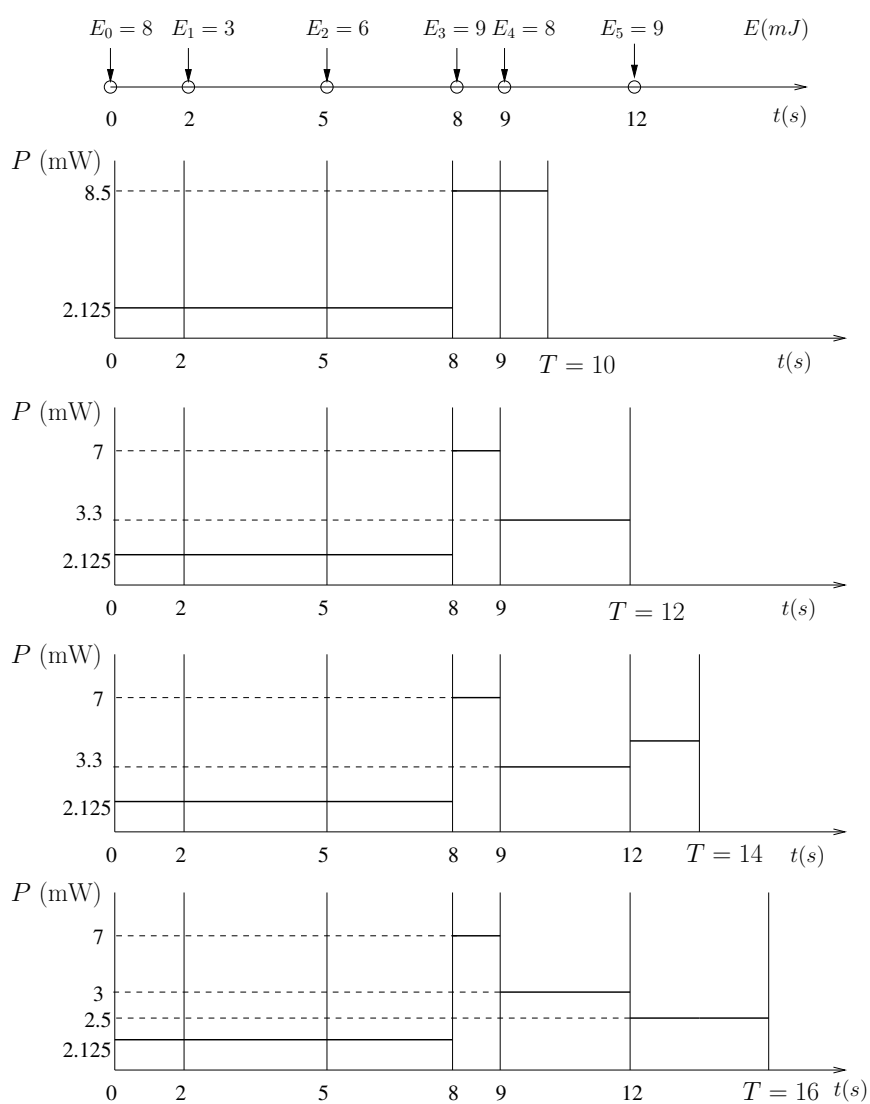

Fig. 4. Energy arrivals occur at $[2,5,8,9,12] \mathrm{s}$ with amounts $[3,6,9,8,9]$ $\mathrm{mJ}$ and the initial energy in the battery at time zero $E_{0}=8 \mathrm{~mJ}$. The optimal transmission policy for $T=10 \mathrm{~s}, T=12 \mathrm{~s}, T=14 \mathrm{~s}$ and $T=16 \mathrm{~s}$.

$E_{1}=3 \mathrm{~mJ}, E_{2}=6 \mathrm{~mJ}, E_{3}=9 \mathrm{~mJ}, E_{4}=8 \mathrm{~mJ}, E_{5}=9$ $\mathrm{mJ}$. The battery energy at time $t=0 \mathrm{~s}$ is $E_{0}=8 \mathrm{~mJ}$. We show the optimal total transmit power sequences for $T=10$ $\mathrm{s}, T=12 \mathrm{~s}, T=14 \mathrm{~s}$ and $T=16 \mathrm{~s}$ in Fig. 4. Initial energy in the battery and the first two energy arrivals are spread till $t=8 \mathrm{~s}$. However, at most $2 \mathrm{~mJ}$ energy can flow from the time interval $[8,9] \mathrm{s}$ to the future as the finite battery constrains the energy flow. For example, for $T=10 \mathrm{~s}$, only $0.5 \mathrm{~mJ}$ energy is transfered from $[8,9] \mathrm{s}$ interval while for $T=12 \mathrm{~s}, 2 \mathrm{~mJ}$ limit is hit and the power in $[8,9] \mathrm{s}$ is kept at $7 \mathrm{~mJ}$ (which leads to $7 \mathrm{~mW}$ power in that interval). Similarly, at most 1 $\mathrm{mJ}$ energy can flow from $[9,12] \mathrm{s}$ interval to the future. This leads to a non-monotonic total transmit power sequence. We plot the resulting maximum departure regions in Fig. 5. Note that the maximum departure regions are strictly convex for all $T$ and monotone in $T$.

\section{CONCLUSIONS}

We studied communication over vector broadcast channels when the transmitter harvests energy from nature. We characterized the trade-off between the performances of the users via the maximum departure region. We proved that the optimal total transmit power policy that achieves the boundary of the maximum departure region is the same as the optimal policy

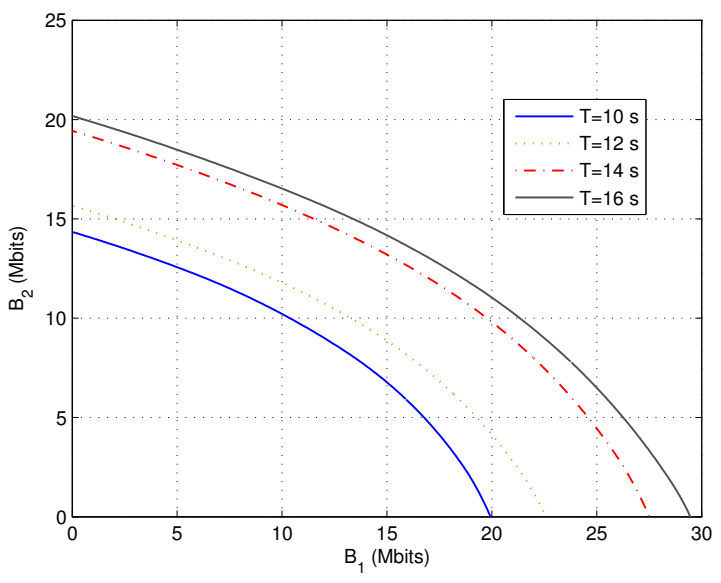

Fig. 5. The maximum departure region for the vector broadcast channel under the given energy arrivals for various $T$.

for the scalar single-user channel. We showed that the optimal total transmit power is found by a directional water-filling algorithm and the optimal splitting of the power among the users and the channels is performed in each epoch separately.

\section{REFERENCES}

[1] J. Lei, R. Yates, and L. Greenstein, "A generic model for optimizing single-hop transmission policy of replenishable sensors," IEEE Trans. Wireless Commun., vol. 8, pp. 547-551, February 2009.

[2] V. Sharma, U. Mukherji, V. Joseph, and S. Gupta, "Optimal energy management policies for energy harvesting sensor nodes," IEEE Trans. Wireless Commun., vol. 9, pp. 1326-1336, April 2010.

[3] C. Ho and R. Zhang, "Optimal energy allocation for wireless communications powered by energy harvesters," in IEEE ISIT, June 2010.

[4] J. Yang and S. Ulukus, "Transmission completion time minimization in an energy harvesting system," in CISS, March 2010.

[5] J. Yang and S. Ulukus, "Optimal packet scheduling in an energy harvesting communication system," IEEE Trans. Comm., to appear. Also available at [arXiv: 1010.1295$]$.

[6] K. Tutuncuoglu and A. Yener, "Optimum transmission policies for battery limited energy harvesting nodes," IEEE Trans. Wireless Comm., submitted, September 2010. Also available at [arXiv:1010.6280].

[7] O. Ozel, K. Tutuncuoglu, J. Yang, S. Ulukus, and A. Yener, "Transmission with energy harvesting nodes in fading wireless channels: Optimal policies," IEEE Journal on Selected Areas in Communications, vol. 29, pp. 1732-1743, September 2011.

[8] J. Yang, O. Ozel, and S. Ulukus, "Broadcasting with an energy harvesting rechargeable transmitter," IEEE Trans. Wireless Comm., submitted, October 2010. Also available at [arXiv:1010.2993].

[9] M. A. Antepli, E. Uysal-Biyikoglu, and H. Erkal, "Optimal packet scheduling on an energy harvesting broadcast link," IEEE Jour. on Selected Areas in Commun., vol. 29, pp. 1721-1731, September 2011.

[10] O. Ozel, J. Yang, and S. Ulukus, "Broadcasting with a battery limited energy harvesting rechargeable transmitter," in WiOpt, May 2011.

[11] O. Ozel, J. Yang, and S. Ulukus, "Optimal broadcast scheduling for an energy harvesting rechargeable transmitter with a finite capacity battery," IEEE Trans. Wireless Comm., submitted, May 2011.

[12] D. N. C. Tse, "Optimal power allocation for parallel gaussian broadcast channels," Technical Report, 1997. Available at www.eecs.berkeley.edu/ dtse/broadcast2.pdf.

[13] L. Li and A. Goldsmith, "Capacity and optimal resource allocation for fading broadcast channels-part i," IEEE Trans. Inform. Theory, vol. 47, pp. 1083-1102, March 2001.

[14] O. Ozel, J. Yang, and S. Ulukus, "Optimal transmission schemes for parallel and fading broadcast channels with an energy harvesting rechargeable transmitter," Elsevier Computer Communications, submitted, September 2011. 